\title{
Optimization of Energy Consumption for Task Scheduling on Uni-Processor and Multiprocessor for Step Topology under Distributed Environment
}

\author{
Kamlesh Kumar Verma \\ Department of Computer \\ Science Babasaheb Bhimrao \\ Ambedkar University \\ Raebareli Road, Vidya Vihar \\ Lucknow 226025(UP) India
}

\author{
Vipin Saxena \\ Department of Computer Science \\ Babasaheb Bhimrao Ambedkar University \\ Raebareli Road, Vidya Vihar \\ Lucknow 226025 (UP) India
}

\begin{abstract}
Distributed computer networking plays a very crucial role in the Business, Industries, Education, Research and Development areas. Many users work on the heterogeneous devices which have different configurations. In distributed network communication takes place from one to one machine, one to many machines or many to one machine. Hence, tasks are migrated from one device to another device which is the important property of the distributed system. Due to rapid increase of the users on the devices connected across the distributed network, the management of the computer networks is a very big and challenging area of research. In the present work, different devices are connected across the step topological networks and an attempt is made to reduce the overall energy consumption when data is flowing from one device to another device. Optimization of energy consumption reduces the overall cost of transfer of data. Multiprocessor and Uni-processor cases are considered in special cases and computed results are represented in the form of tables. A well known Hungarian methodology is used for optimization of the overall energy.
\end{abstract}

\section{Keywords}

Distributed Network, Step Topology, Lagrangian Method, Hungarian Method, Uni-Processor, Multiprocessor, Energy Optimization.

\section{INTRODUCTION}

In the current scenario, distributed computing plays a vital role in computing. It takes an edge over the centralized computing system due to the low cost and higher efficiency. Multiple windows under the excellent operating system environment may appear on the computer screen for the multiple computing purposes. Nowadays personal computers may have more than one processor due to exhaustive research available for dual core processors or even multiprocessors when arranged via a static network topology which should be efficient and low cost involvement. Further, a task is defined a piece of macro, process, subroutine, subprogram, etc. and for multiple tasks scheduling algorithms are applicable for completion of tasks within minimum time. Since all the tasks are executed on the processor taking either uni-processor or multi-processor, therefore, energy optimization is a big challenge for the resources. One can say that the energy can be consumed when task is executed on the uni-processor or multi-processors connected across the step network. Electrical energy may be consumed by the hard disk, processors, motherboard, power supply etc. which is further categorized as a fixed energy and variable energy. Due to several advantages of distributed computing over the centralized computing, slowly-slowly, many organizations are shifting their computing labs from centralized system to distributed system. In this computing is a collection of various computer networks incorporated with a single server or computers. The computers are retrieving information with each other related computers. In this computing all computers are connected in common component computer devices, for the operation of various computing operation. All the computer components are initially worked as purely on scheduling basis as synchronize and asynchronies. The process may be FCFS (First come first serve), other job scheduling algorithm and Round-Robin is one of the most popular approach of the scheduling of the tasks. The computing component consumes the electric energy component wise, like chipset mother board, central processing unit, electromechanical devices (i.e. Fan), Hard disk. All the component devices consume more energy during action performance. The electric energy increases by the work load and it is a continuous process of synchronization if all components are busy. The electric energy reduces the cost which may be lack of workload during sleep mode and due to absence of data packets. There are two methods used for the reduction of the energy consumption. Firstly the component in sleep mode operation and secondly the rate of network operation to be applied through workload. In this work, authors have attempted to optimize consumed energy on the Uni-processor as well as the multiprocessors for the task scheduling. Standard specification of Uni-processor and multiprocessors are considered consumed energy is computed. Energy of a system is computed for Uni-processor and multi-processor using Lagrangian multipliers with resource tasks and processes. For optimizing the energy consumption, a well known Hungarian method is used.

\section{RELATED WORK}

Pecero $S^{\prime}$ anchez et al. [1] have given a scheduling algorithm for reducing energy consumption by dynamic voltage scaling (DVS). In this algorithm, authors found minimum time to finish complete process in scheduling. Wang et al [2] have described an algorithm for energy optimization method. Authors used dynamic reconfiguration in real time scheduling for multitasking systems. Authors used flexible parameter based on dynamic reconfiguration for completion of processes in multitasking system. Wang et al [3] have focused for solving the energy optimization problem for real time multiprocessor systems in streaming pipelining with dynamic voltage scaling and dynamic power management in complete transition process. A scheduling genetic algorithm is proposed for best scheduling process. By the result of proposed 
technique, $24.4 \%$ reduction in energy consumption was observed. Chen et al. [4] have used a mix integer linear programming technique. In this method, they have used the combined Dynamic Voltage Frequency Scaling (DVFS) and Dynamic Power Management (DPM) for the reduction of energy consumption in Uni-processor systems. The DVFS is constraints with voltage and frequency scaling at the time of processes scheduling and DPM is defined as the power constraints of various hardware equipments. Anne et al. [5] have invented a classical scheduling algorithm for unified scheduling process. This scheduling process is depends on probability, offline for energy consumption. Zhang et al. [6] have predicted a scheme based packet scheduling in Ethernet passive optical network, authors used an algorithm and discussed sleep based model for energy saving approaches. Authors have also interpreted the energy consumption on sleeping and low loaded networks unit. Anbazhagan et al. [7] have investigated power management mechanisms for power saving in heterogeneous traffic based on Combined Cyclic Exponent (CCE), Combined Anti-Cyclic Binary Exponent (CABE) and Combined Truncated Binary Exponent (CTBE) algorithms based in different time interval. Kiani et al [8] have given a theory of real time tasks scheduling. This scheduling process is based on CPU and read write tasks of hard disk. The execution time of the process of the CPU and read write time of hard disk without tasks missing. Yan et al. [9] have explained a numerical study of energy consumption which depends on time efficiency with five structural topologies and four different routing methods i.e. (i) ER Random Graph Model (ii) Kleinberg Small World Model (iii) Scale Free Model (iv) Grid Model. Although different devices have a range of power consumption but due to heavy load across the network, overall time of execution increases and power is directly related to the time, therefore energy consumption increases. Various authors have used the Mathematical techniques to optimize the energy consumptions and some of the important references are described.. Zhang et al. [10] have given a green task scheduling algorithms for heterogeneous computer networks. In these algorithms, the process scheduling with continuous speeds and discrete speeds for the energy consumption are described. Authors have considered two cases (i) Heuristically Assigning Tasks (ii) Minimum Optimal Speeds for all tasks assigned to each computer. The algorithm is used for energy efficient equipment as mobile devices, sensor networks; data centre, and distributed computing networks. Rehaiem et al. [11] have discovered a scheduling priority based algorithms of real time scheduling for embedded systems. In this method, authors used embedded systems with neural networks for low power consumption optimization. The combination of the DVS and neural Feedback scheduling process are used for energy optimization. Boiardi et al. [12] have described the joint Mathematical planning and energy management operation for energy consumption. They have discussed a Mathematical framework and designing the network for optimizing the power consumption. Zhang et al. [13] have given an algorithm named as Shuffled Frog Leaping Algorithm for real time tasks scheduling in heterogeneous networks. This algorithm is used in multiprocessor systems in the time of process scheduling. Rituraj et al.[14] have described three schemes for Orthogonal Frequency Division Multiple Access (OFDMA) named as Hungarian method, Game Theory and Gale-Shapley matching scheme for finding the energy performance. Li et al. [15] have described the power allocation and task scheduling process on multiprocessor computers with energy and time relations. In their work, authors discussed how the energy in multiprocessor affects with the time relation constraints. Jena et al. [16] have focused a theory on task scheduling using a multi objective optimization method for energy consumption with the related of processing time. Da-Ren et al. [17] have invented a model of variable voltage depend processor with the discrete time voltage/speed. The DVFS scheduling can be defined in two steps (i) inter-task DVFS (ii) intra-task DVFS. The DVFS scheduling technique is a most effective for reducing the power consumption. Bharti et al. [18] have given a Task Requirements Aware Pre-processing and Scheduling (TRAPS) for CPU. The scheduling of processor is measured by the incoming tasks. Proposed energy monitoring system works on reducing message process and energy consumption. Sousa et al. [19] have given an algorithm based on Meta heuristic technique for energy resource scheduling in smart grids. Zhao et al. [20] have discovered a task scheduling method for datasets and tasks. In this method there are two conditions (i) datasets of task scheduling (ii) tree to tree task scheduling. Ismail et al. [21] have given a model based technique named as Energy Aware Tasks Scheduling for the tasks scheduling. The application of Energy Aware Tasks Scheduling model increases the efficiency and reduces the energy consumption. The consumption of energy is depending on work load of data servers. C. Douligeris and G. Feng [25] have proposed a theory "guided trial-and-error" method for energy optimization. Authors have solved the technique by computer simulation on the assignment problem and N-Queen problem of different aspects.

\section{BACKGROUND}

\subsection{Distributed Computing Networks}

The distributed computing network is an interconnected network over various resources like laptop, printers, mobile, desktop, and other peripheral devices connected through a server. The devices may communicate from one to one and many to one device for applications. The network peripheral devices may send the process application for retrieving the information via server application as per instruction sequencing process.

\subsection{Step Distributed Networks}

In this network, there are at least three nodes linking on the network and communicated in distributed manner. When it is extended towards step network, there are many computers connected through a server for various application operations. In this network beneficial as resource sharing, scalability, robustness, performance speed and cost are important factors of study. It is challenging task for step network like as latency, synchronization process and failure data transmission, security, transparency and scalability. A view of step network is shown below in figure 1 .

\subsection{Energy Optimization Methods}

\subsubsection{Dynamic Voltage Frequency Scaling (DVFS) Method}

It is a very crucial technique used for reducing the energy consumption. If the voltage $\mathrm{V}$ decreases to the system then energy and frequency will also getting low. If the voltage increases of power supply then the system increases the frequency and generating power. The dynamic voltage scaling technique is used for reducing the energy efficiency. In the computer architecture, the voltage used in a device wise component is increased or decreased, depending upon conditions. So it is beneficial for voltage balancing to the networks system. Voltage measurement is important for energy reducing very low values when operating at low speeds. The computation of voltage is done by [3-4] 


$$
V=\sqrt{\frac{P}{C \cdot f}}
$$

Where, $\mathrm{P}, \mathrm{C}$ and $\mathrm{f}$ are power, capacitance and frequency respectively. DFS can be used for energy conservation for lowering the heat produced by a processor by lowering frequency. The low frequency will increase the amount of time and a processing element needs to complete a task. Energy is conserved with DFS because the Peripheral Equipment (PE) like Motherboard, Hard disk, CPU and other electromechanical components (i.e. fan) consumes less power when running at low frequency. The frequency is given by.

$$
f=\frac{P}{C V^{2}}
$$

\subsubsection{Clock Gating}

Clock gating is a third technique of energy optimization methodology commonly used to conserve energy on the processing element level. Clock gating adds additional logic to the processing elements. This certain parts of the switching action from changing states. This method reduces the power of processing elements from constantly switching which accounts for a large fraction of the processing elements. Clock gating reduces the unwanted switching on the parts of clock net by disabling the clock.

\subsubsection{Routing Techniques}

\subsubsection{Link State Routing}

The link state routing is a state description routing as the data transmission flow in one node to other neighbour node. In this method, routing for computation of shortest path is done by Dijkstra's Algorithm from a node to all other nodes. The Dijkstra's algorithm computes the end to end routes, and finds the shortest path of the routing store to the next node address. This method is also used for reducing the energy consumption. This routing technique defines states of node and used as linking state according to the process time scheduling in network topology. Routing used in this is as the scheduling constraints, which is minimum shortest task scheduling.

\subsubsection{Distance Vector Routing}

Another method is a distance-vector routing which defines the length of the shortest-path from each of its neighbour's node to every destination in the network, and uses information status to measure its own distance and next successor router (r) to each destination. Each update message sent by a router to its neighbours contains a vector with one or more, each of which specifies as a minimum, the distance to a given destination's, each router sends only described information data flow. In this routing, the process scheduling is based on spanning distance. The spanning distance defines as the minimum shortest distance covered by the node vector.

\section{RESEARCH METHODOLOGY}

In the proposed model, different nodes which are arranged through the step topological structure as shown in figure1 make service requests which keep in the service queues. Let us consider each node is evaluated with the Uni-processor, computer with time constraint $\mathrm{T}$ and energy Constraint $\mathrm{E}$. The transmission time for $\mathrm{n}$ nodes is represented by matrix $\mathrm{Ti}, \mathrm{j}$ since all nodes are autonomous collection to form distributed system then ri $(\mathrm{i}=1 \ldots \mathrm{n})$ represents of task scheduling i \& sum of all ri is $\mathrm{R}$, pi represents power allocated to each task scheduling i in terms of supply voltage, clock frequency, etc. Then execution speed of task $\mathrm{i}, \mathrm{si}=\mathrm{pi}$ $(1 / \alpha)$, where $\alpha=1+2 / \varphi \geq 3,0<\varphi \leq 1$, and $\varphi$ is the linear change in supply voltage, and the energy consumption of a particular resource is given by [26]. Energy $\alpha$ Power input $\times$ Amount of workdone for Task $(i)$

$$
E=r_{i}\left(p_{i}\right)^{1-\frac{1}{\alpha}}
$$

Then total energy consumption of resources is given by [26],

$$
\begin{gathered}
E\left(p_{1}, p_{2} \ldots \ldots \ldots \ldots p_{n}\right)=r_{1} p_{1}^{1-\frac{1}{\alpha}}+r_{2} p_{2}^{1-\frac{1}{\alpha}}+\ldots \ldots \ldots \ldots \ldots . r_{n} p_{n}^{1-\frac{1}{\alpha}} \\
=\sum_{i=1}^{n} r_{n} p_{n}^{1-\frac{1}{\alpha}}
\end{gathered}
$$

We should minimize E (p1, p2 .............pn) but subject to time constraints and let schedule length $\mathrm{t} 1, \mathrm{t} 2$..........tn does not exceed $\mathrm{T}$ then, let a function is considered on variables( $\mathrm{p} 1$, $\mathrm{p} 2$............pn) and is given as $\mathrm{F}(\mathrm{p} 1, \mathrm{p} 2$............pn), which are less than of total time scheduling $\mathrm{T}$ given by [26].

$$
\begin{aligned}
F\left(p_{1}, p_{2} \ldots \ldots \ldots . . p_{n}\right)= & \frac{r_{1}}{p_{1}^{1 / \alpha}}+\frac{r_{2}}{p_{2}^{1 / \alpha}} \ldots \ldots \ldots \ldots \ldots . . . \frac{r_{n}}{p_{n}^{1 / \alpha}} \leq T \\
& \sum_{i=1}^{n} \frac{r_{n}}{p_{n}^{1 / \alpha}} \leq \boldsymbol{T}
\end{aligned}
$$

Now, Let $\square \mathrm{i}$ is Lagrangian multiplier formulated and then it is given by [26]

$$
\begin{gathered}
\lambda_{i}=\frac{\nabla E}{\nabla F} \\
\nabla E=\lambda_{i} \nabla F \\
\frac{\partial E}{\partial p_{i}}=\lambda_{i} \frac{\partial F}{\partial p_{i}}
\end{gathered}
$$

From equation (3) and equation (5) given by [26], where $\lambda_{i}=$ $\mathrm{p}_{\mathrm{i}}(1-\alpha)$, for all $1 \leq \mathrm{i} \leq \mathrm{n}$.

$$
\begin{gathered}
r_{i}\left(1-\frac{1}{\alpha}\right) \frac{1}{p_{i}^{1 / \alpha}}=\lambda_{i} r_{i}\left(-\frac{1}{\alpha}\right) \frac{1}{p_{i}^{1+1 / \alpha}} \\
1-\frac{1}{\alpha}=-\frac{\lambda_{i}}{\alpha p_{i}} \\
p_{i}=\frac{\lambda_{i}}{(1-\alpha)} \\
E_{i}=\frac{R_{i}^{\alpha}}{T^{(\alpha-1)}} \\
R_{i}^{\alpha}=E_{i} T^{(\alpha-1)} \\
R_{i}=E_{i}^{\frac{1}{\alpha}} T^{\frac{(\alpha-1)}{\alpha}} \\
E_{i}=\frac{R_{i}^{\alpha}}{T^{(\alpha-1)}} \\
R_{i}=\left(E_{i}\right)^{\frac{1}{\alpha}} T^{\left(1-\frac{1}{\alpha}\right)}
\end{gathered}
$$




$$
\begin{gathered}
E=\sum_{i=1}^{n} r_{i}\left(\frac{\lambda_{i}}{1-\alpha}\right)^{1-1 / \alpha} \\
R=r_{1}+r_{2}+\ldots \ldots . . .+r_{n} \\
R=\sum_{i}^{n} r_{i} \\
T=\left(p_{i}\right)^{1 / \alpha} / R
\end{gathered}
$$

Where $\mathrm{R}$ is the total Task scheduling of requirement of the $\mathrm{n}$ tasks for resources, In the case of multiprocessor, the energy consumption is given by,

$$
R=E_{i}^{\frac{1}{\alpha}} \times T^{(1-1 / \alpha)}
$$

The above formula used for multiprocessor for finding the energy for different resources.

\section{PROPOSED WORK}

In the present work, the following cases are described below based on the above formulae

\subsection{Uni-processor Case}

A Uni-processor system has a single computer processor, which is used to execute many tasks. All the tasks are related to a single processor and also to various operations controlled by the single processor computer. The power supply of the Uni-processor does not change and remains at fixed time by the component peripherals. The physical limitations of single processor are limiting controlling tasks. Let the coefficient $\alpha$ $=3,4,5$ then Lagrangian multiplier $\lambda_{\mathrm{I}}$ is calculated by the equation 7 , for the uni-processor as given by [26]

$$
\lambda_{i}=p_{i}(1-\alpha)
$$

Where $\lambda_{i}$ is the Lagrangian Multiplier and processes $p_{i}$ are different tasks scheduling and power supply is taken as uniform by the equation 8 .

Table 2. Computation of energy consumption for Uni-processor $(\alpha=3,4,5)$

\begin{tabular}{llccccccc}
\hline SI. No. & $\begin{array}{c}\text { Power } \\
\left(\mathbf{p}_{\mathbf{i}}\right)\end{array}$ & Tasks & $\begin{array}{c}\text { Lagrangian } \\
\text { Multiplier } \\
\text { for }(\alpha=3)\end{array}$ & $\begin{array}{c}\text { Energy }\left(\mathbf{e}_{\mathbf{i}}\right) \\
\text { Consumption } \\
\text { for }(\alpha=3)\end{array}$ & $\begin{array}{c}\text { Lagrangian } \\
\text { Multiplier } \\
\text { for }(\alpha=4)\end{array}$ & $\begin{array}{c}\text { Energy }\left(\mathbf{e}_{i}\right) \\
\text { Consumption } \\
(\alpha=4)\end{array}$ & $\begin{array}{c}\text { Lagrangian } \\
\text { Multiplier } \\
\text { for }(\alpha=5)\end{array}$ & $\begin{array}{c}\text { Energy }\left(\mathbf{e}_{i}\right) \\
\text { Consumption } \\
\text { for }(\alpha=5)\end{array}$ \\
\hline 1. & $\mathrm{p}_{1}$ & 100 & 0.0052 & 1.80 & 0.0030 & 0.560 & 0.0025 & 0.270 \\
2. & $\mathrm{p}_{2}$ & 105 & 0.0047 & 1.81 & 0.0031 & 0.588 & 0.0023 & 1.680 \\
3. & $\mathrm{p}_{3}$ & 110 & 0.0045 & 1.76 & 0.0030 & 0.616 & 0.0027 & 0.220 \\
4. & $\mathrm{p}_{4}$ & 115 & 0.0043 & 1.84 & 0.0031 & 0.644 & 0.0021 & 0.260 \\
5. & $\mathrm{p}_{5}$ & 120 & 0.0041 & 1.86 & 0.0027 & 3.480 & 0.0020 & 2.640 \\
6. & $\mathrm{p}_{6}$ & 125 & 0.0046 & 1.93 & 0.0026 & 0.587 & 0.0019 & 2.610 \\
7. & $\mathrm{p}_{7}$ & 130 & 0.0038 & 2.07 & 0.0025 & 0.611 & 0.0019 & 0.247 \\
8. & $\mathrm{p}_{8}$ & 135 & 0.0037 & 2.02 & 0.0024 & 3.51 & 0.0018 & 0.0675 \\
\hline
\end{tabular}

For the Uni-processor, the following devices are considered with standard specification configuration. Now the energy computation with the different resource scheduling $r_{i}$ is considered with power supply and standard specification of component wise, the standard power supply max values is taken. Let $\mathrm{r}_{1}, \mathrm{r}_{2}, \mathrm{r}_{3}, \mathrm{r}_{4}, \mathrm{r}_{5}, \mathrm{r}_{6}, \mathrm{r}_{7}$ and $\mathrm{r}_{8}$ are the various tasks scheduling for the multiprocessor component devices. Let $r_{1}$ $=100, r_{2}=105, r_{3}=110, r_{4}=115, r_{5}=120, r_{6}=125, r_{7}=130$ and $r_{8}=135$ at different state when information flow from on multi-core shared memory. The formula used for finding

$$
E=\sum r_{i}\left(\frac{\lambda_{i}}{1-\alpha}\right)^{1-\frac{1}{\alpha}}
$$

The energy consumption of the Uni-processor can be obtained by putting the value of Lagrangian multiplier and coefficient $\alpha$ in above formula. The energy consumption is calculated by Lagrangian values in different task scheduling with the varying $\alpha$ values, simultaneously. The energy consumption of the Uni-processor with the Lagrangian multiplier and coefficient $\lambda$ are demonstrated based on Lagrangian values through different resource tasks scheduling with $\alpha=3,4,5$.

Table 1.Uni-processor related with various component wise Specification.

\section{Table 1. Uni-processor related with various component wise Specification}

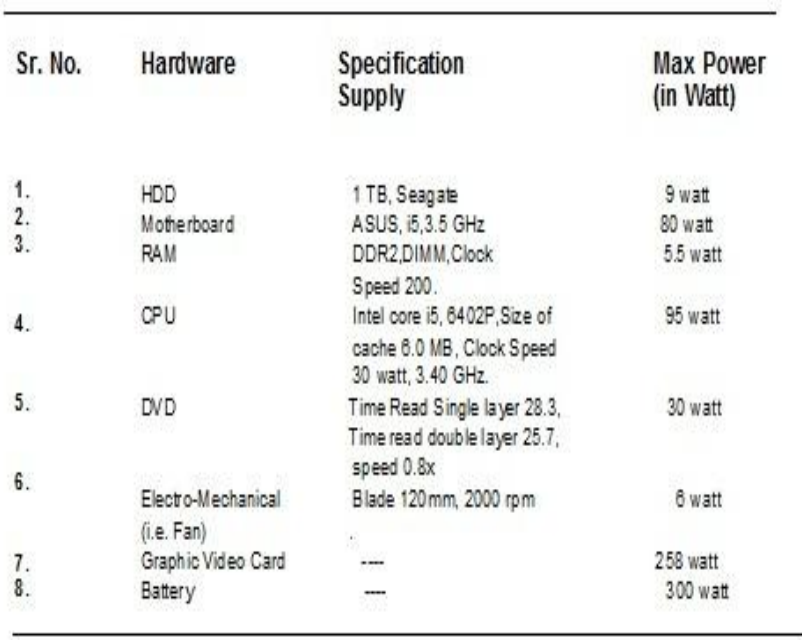

energy for each component based with different task is given by equation (7). The following matrix represented in the form of table is designed for the eight resources by taking combinations in the form of $8 \times 8$ (Ei X Ri) [26].

$$
E=\sum p_{i}{ }^{1-\frac{1}{\alpha}} \times r_{i}
$$

To compute the optimal result, a well-known Hungarian method is used and final matrix is given below by the following steps, 
Draw lines through appropriate rows and columns so that all the zero entries of the cost matrix are Covered and the minimum number of such lines is used and optimize the above matrix, a well-known Hungarian method [14] is used and final matrix is given below, The formula is used for finding the energy , where pi is power of hardware device $E=(9)^{2 / 3} \times$ $100=426.3$ Joule and so on,

\begin{tabular}{|l|l|l|l|c|c|c|c|}
\hline 426 & 652 & $\underline{\mathbf{5 4 4}}$ & 567 & 610 & 763 & 697 & 479 \\
\hline 1851 & 2824 & 2353 & 2447 & $\underline{\mathbf{2 6 3 6}}$ & 3314 & 3012 & 2071 \\
\hline 2075 & $\underline{\mathbf{3 1 6 9}}$ & 2641 & 2746 & 2958 & 3698 & 3381 & 2354 \\
\hline$\underline{\mathbf{3 1 3}}$ & 470 & 392 & 417 & 439 & 548 & 501 & 345 \\
\hline 4128 & 3192 & 5160 & 5366 & 5779 & 7224 & $\underline{\mathbf{6 6 0 5}}$ & 4541 \\
\hline 976 & 1464 & 1220 & $\underline{\mathbf{1 2 6 8}}$ & 1366 & 1708 & 1562 & 1074 \\
\hline 136 & 498 & 415 & 431 & 465 & 581 & 531 & $\underline{\mathbf{3 6 5}}$ \\
\hline 927 & 6850 & 5708 & 5937 & 6394 & $\underline{\mathbf{7 9 9 2}}$ & 7307 & 5024 \\
\hline
\end{tabular}

\begin{tabular}{|c|c|c|c|c|c|c|c|}
\hline 36 & 201 & $\underline{\mathbf{0}}$ & 0 & 55 & 921 & 368 & 15 \\
\hline 582 & 0 & 162 & 91 & $\underline{\mathbf{0}}$ & 341 & 24 & 394 \\
\hline 703 & $\underline{\mathbf{0}}$ & 219 & 137 & 23 & 302 & 0 & 456 \\
\hline$\underline{\mathbf{0}}$ & 234 & 3 & 1 & 77 & 987 & 415 & 0 \\
\hline 1874 & 3201 & 924 & 741 & 426 & 0 & $\underline{\mathbf{0}}$ & 1493 \\
\hline 187 & 90 & 25 & $\underline{\mathbf{0}}$ & 0 & 677 & 204 & 121 \\
\hline 197 & 226 & 0 & 7 & 71 & 974 & 405 & $\underline{\mathbf{0}}$ \\
\hline 5843 & 311 & 1144 & 938 & 579 & $\mathbf{0}$ & 66 & 1778 \\
\hline
\end{tabular}

The optimal values of Uni-processor represented as underlined, measured by the Hungarian Method, and the optimized value of energy consumption is given by [14]

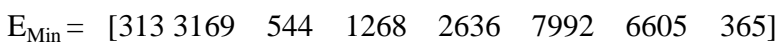

$$
\mathrm{E}_{\mathrm{Min}}=22892 \mathrm{KJ} \text {. }
$$

The relationship between energy and resource is also shown below in figure 2 .

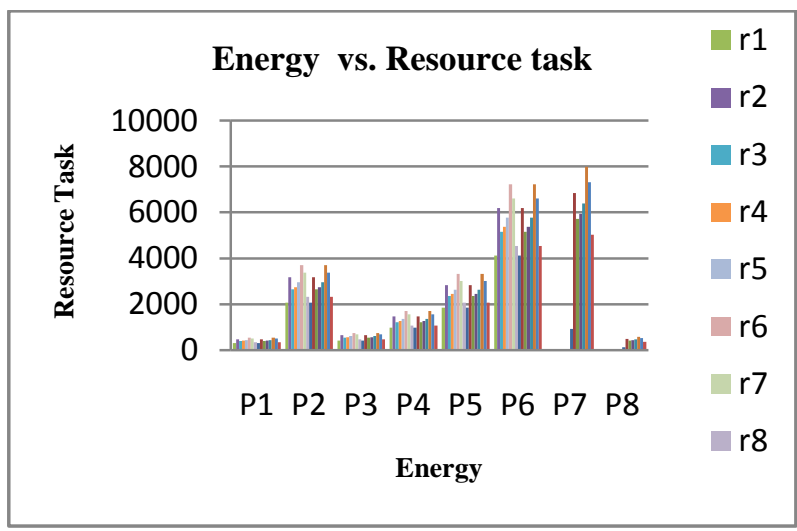

\section{Figure 2. Relationship between Energy and Resource Task in a Uni-Processor}

In Uni-processor, there is different component-wise power supply; the power supply is fixed with maximum value in unit emitted by each component. The different resource aspect task scheduling is computed for finding the energy consumption with different tasks; energy is computed at different task scheduling. One by one, each component has been computed by the estimation process with different interval task scheduling. The energy optimal paths are computed above and overall energy is optimized by Hungarian optimization method i.e. $22892 \mathrm{KJ}$

\subsection{Multiprocessor Case}

In the multiprocessor system, the use of two or more central processing units (CPU's) within a single computer system is available. The multiprocessor system has better performance in terms of cost and tasks scheduling. It's also called multi core system with each one is sharing the common main memory as well as the peripherals. It has better throughput, hardware sharing within processors. Energy consumption parameters for multiprocessor system are Task Time Scheduling (ms), Input Power Supplying and Energy-Delay (ED). Let us consider the execution time of each process as $\mathrm{T}=10$ milliseconds in multiprocessor and the execution on different task $\mathrm{R}$ is to be computed by the equation 11 and computed resources for eight processes are also demonstrated column-wise results as given below:

$R_{1}=\left[\begin{array}{l}36 \\ 33 \\ 62 \\ 34 \\ 51 \\ 65 \\ 89 \\ 92\end{array}\right]$
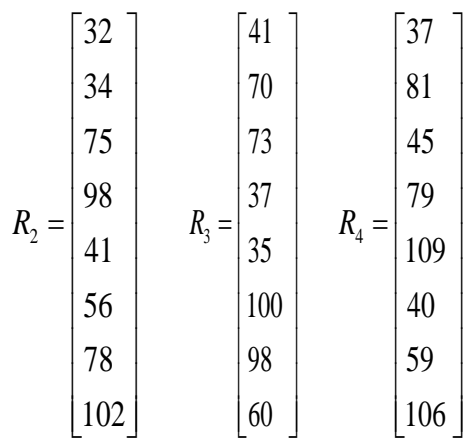

$R_{5}=\left[\begin{array}{l}66 \\ 65 \\ 39 \\ 47 \\ 84 \\ 88 \\ 118 \\ 119\end{array}\right]$

$$
R_{6}=\left[\begin{array}{l}
52 \\
77 \\
74 \\
65 \\
58 \\
46 \\
101 \\
48
\end{array}\right]
$$

$$
R_{7}=\left[\begin{array}{l}
84 \\
51 \\
55 \\
71 \\
107 \\
100 \\
89 \\
52
\end{array}\right]
$$$$
R_{8}=\left[\begin{array}{l}
46 \\
82 \\
71 \\
53 \\
85 \\
99 \\
48 \\
57
\end{array}\right]
$$

The relationship matrix with different resources allocation $\mathrm{R}$ power supply and energy between each component wise $E_{i}$ with same time for multiprocessor across the step distribution network is given below in $8 \times 8\left(\mathrm{E}_{\mathrm{i}} \times \mathrm{R}_{\mathrm{i}}\right)$.

\begin{tabular}{|l|l|c|c|l|l|l|l|}
\hline 36 & 33 & 62 & $\underline{\mathbf{3 4}}$ & 51 & 65 & 89 & 92 \\
\hline 32 & $\underline{\mathbf{3}} \mathbf{4}$ & 75 & 98 & 41 & 56 & 78 & 102 \\
\hline 41 & $\mathbf{7 0}$ & 73 & 37 & $\underline{\mathbf{3 5}}$ & 100 & 98 & 60 \\
\hline$\underline{\mathbf{3 7}}$ & 81 & 45 & 79 & 109 & 40 & 59 & 106 \\
\hline 66 & 65 & $\underline{\mathbf{3 9}}$ & 47 & 84 & 88 & 118 & 119 \\
\hline 52 & 77 & 74 & 65 & 58 & $\underline{\mathbf{4 6}}$ & 101 & 48 \\
\hline 84 & 51 & 55 & 71 & 107 & 100 & 89 & $\underline{\mathbf{5 2}}$ \\
\hline 46 & 82 & 71 & 53 & 85 & 99 & $\underline{\mathbf{4 8}}$ & 57 \\
\hline
\end{tabular}


The above matrix is further optimized by the use of Hungarian Method and 0 values represent the optimized cell.

\begin{tabular}{|c|c|c|c|c|c|c|c|}
\hline 5 & 0 & 29 & $\underline{\mathbf{0}}$ & 18 & 32 & 54 & 58 \\
\hline 0 & $\underline{\mathbf{0}}$ & 41 & 63 & 7 & 22 & 42 & 67 \\
\hline 8 & 35 & 38 & 1 & $\underline{\mathbf{0}}$ & 65 & 61 & 24 \\
\hline$\underline{\mathbf{0}}$ & 42 & 6 & 39 & 70 & 1 & 18 & 66 \\
\hline 29 & 26 & $\underline{\mathbf{0}}$ & 7 & 45 & 49 & 77 & 79 \\
\hline 8 & 31 & 28 & 18 & 12 & $\underline{\mathbf{0}}$ & 53 & 1 \\
\hline 35 & 0 & 4 & 19 & 56 & 49 & 36 & $\underline{\mathbf{0}}$ \\
\hline 2 & 36 & 25 & 6 & 39 & 53 & $\underline{\mathbf{0}}$ & 10 \\
\hline
\end{tabular}

The optimal values of the above matrix are recorded below:

$$
\begin{gathered}
\mathrm{E}_{\mathrm{Min}}=\left[\begin{array}{cccccccc}
37 & 34 & 39 & 34 & 35 & 46 & 48 & 52
\end{array}\right] \\
\mathrm{E}_{\mathrm{Min}}=325 \mathrm{~J}
\end{gathered}
$$

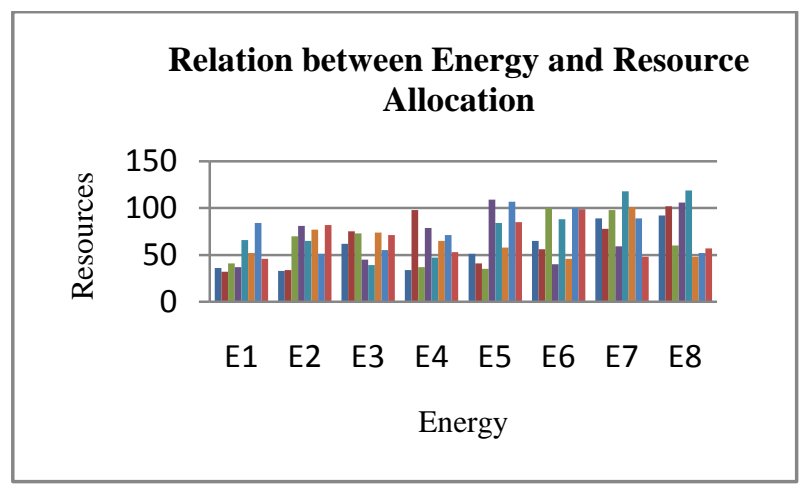

Figure 3. Energy optimization based on various resources components in multiprocessor

A relationship between resource and energy is also shown above in figure 3. In multiprocessor, there is low energy consumption used for step topology network in comparison of Uni-processor case. There are three nodes and energy is calculated for each node. The local power management has each and every component; the local maximum power supply by the component is stable and does not change. The comparison for energy consumption between Uni-processor and multiprocessor is shown below in figure 4 .

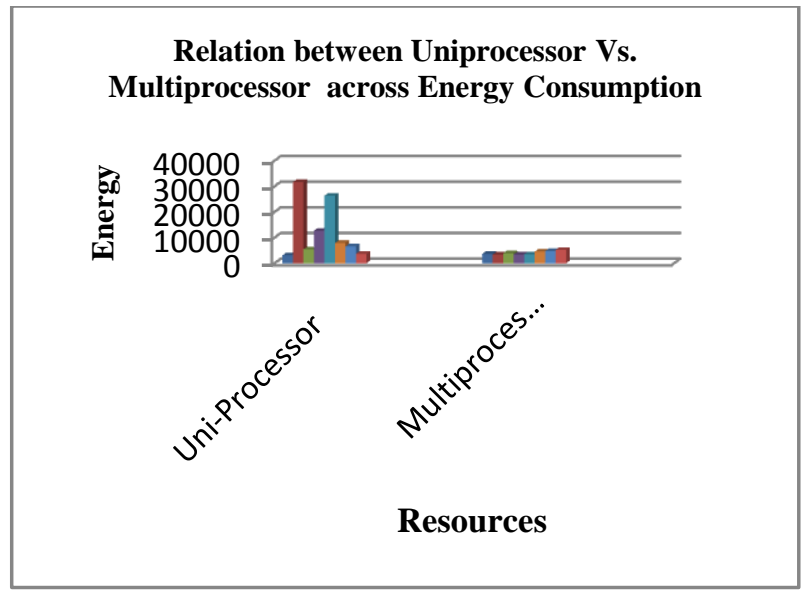

Figure 4. Comparison of Uni-processor and Multiprocessor with Energy Consumption Matrices

\section{CONCLUSION}

In this paper,a model for energy optimization for step network defines the power measurement in distribution computing environment. When information is transferred from one device to another device there are two cases arises one for Uni-processor and another for multiprocessor. The power measurement of the Uni-processor reduces the power with the use of different power allocation by components. The optimal values are given by Hungarian and Lagrangian methods. The power measurement in multiprocessor is also computed with different resource allocations at same time and also for different time interval further the optimal values are observed which are measured through Hungarian Method. If one compares the energy consumption between Uni-processor and multiprocessor. It is further observed that energy is optimized in case of multiprocessor in comparison of Uni-processor.

\section{REFERENCES}

[1] Pecero S'anchez, J.E., Bouvry, P., and Barrios, Hernandez, C.J., Low Energy and High Performance Scheduling on Scalable Computing Systems.2005; 0108 .

[2] Wang,Weixum., Ranka,Sanjay., and Mishra,Prabhat., Energy-aware dynamic reconfiguration Algorithms for real-time multitasking systems. Sustainable Computing: Information and Systems, 2010;(1):35-45.

[3] Wang, YI., Liu, Hui., Liu, Duo., Qin, Zhiwei., Shao, Zili., and Sha Edwin, H-M., Overhead-Aware Energy Optimization for Real-Time Streaming Applications on Multiprocessor System-on- Chip, ACM Transactions on Design Automation of Electronic Systems, 2011; Vol. (16):, No. 2,01-32.

[4] Chen, Gang., Huang, Kai., and Knoli, Alois., Energy Optimization for Real-Time Multiprocessor System- onChip with Optimal DVFS and DPM Combination. ACM Transactions on Embedded Computing Systems, 2013; 01-21.

[5] Anne, Naveen., and Muthu kumar, Venkatesan., Energy Aware Scheduling of A periodic Real-Time Tasks on Multiprocessor Systems. Journal of Computing Science and Engineering, 2013; Vol. (7):No.1, pp. 30-43.

[6] Zhang Luna, Mingyi.,Li, Keqin., Chia-Tien,LoDan.,and Zhang,Yanqing., Energy-Efficient Task Scheduling Algorithms on Heterogeneous Computers with 
Continuous and Discrete Speeds, Sustainable Computing: Informatics and Systems.2013; (3): 109118.

[7] Anbazhagan, Rajesh., and Rangaswamy, Nakkeeran., Investigations on Enhanced Power Saving Mechanism for IEEE $802.16 \mathrm{~m}$ Network with Heterogeneous Traffic. Journal of Network and Computer Applications, 2014; S1084-8045 (14): 48-4.

[8] Kiani Vahdaneh, Mohseni., Zeynab, Rahmani., and Amir, Masoud., Real Time Scheduling for CPU and Hard Disk Requirements-Based Periodic Task with the Aim of Minimizing Energy Consumption, International Journal Information Technology and Computer Science, $2015,10,54-60$.

[9] Yan,Fan., K.H Yeung, Alan., Chen, Guanrong., A Numerical Study of Energy Consumption and Time Efficiency of Sensor Networks with Different Structural Topologies a Routing Method. Communication Nonlinear Science Number Simulation. 2013; (18):25152526.

[10] Zhang, Luna Mingyi., Li, Keqin., Chia-Tien, Lo Dan., Zhang, Yanqing., Energy-Efficient Task Scheduling Algorithms on Heterogeneous Computers with Continuous and Discrete Speeds, Sustainable Computing: Informatics and Systems, 2013; (3):109118.

[11] Rehaiem, G.,Gharsellaou, H., and Ahmed, S. Ben., RealTime Scheduling Approach of Reconfigurable Embedded Systems Based On Neural Networks with Minimization of Power Consumption, IFAC- Papers online, 2016; (49-12):1827-1831.

[12] Boiardi, Silvia.,Capone, Antonio., and Sansó, Brunilde., Joint Design and Management of Energy- Aware Mesh Networks. Ad Hoc Networks. 2012; (10):14821496.

[13] Zhang, Weizhe.,Bai, Enci.,He Hui and M.K. Cheng Albert, Solving Energy-Aware Real-Time Tasks Scheduling Problem with Shuffled Frog Leaping Algorithm on Heterogeneous Platforms, 2013; (15) 13778-13804.

[14] Rituraj and Jagannatham Aditya. K., Optimal Cluster Head Selection Schemes for Hierarchical OFDMA Based Video Sensor Networks. IFIP WMNC'13, 978-14673-5616-9/13, 2013 IEEE.

[15] Li,Keqin.,Energy and Time Constrained Task Scheduling on Multiprocessor Computers with Discrete Speed
Levels, J. Parallel Distributed Computing, 2016; (95):15-28.

[16] Jena, R K., Multi objective Task Scheduling in Cloud Environment Using Nested PSO Framework, Procedia Computer Science, 2015; (57):1219-1227.

[17] Da-Ren,Chen.,Young-Long,Chen.,andYou-Shyang, Chen., Time and Energy Efficient DVS Scheduling for Real-Time Pinwheel Tasks, Journal of Applied Research and Technology, 2014; Vol. (12): 1025-1039.

[18] Bharti, Sourabh., and Pattanaik, K.K., Task requirement aware pre-processing and Scheduling for IoT Sensory environments, Ad Hoc Networks. 2016; (50): 102-114.

[19] Sousa,Tiago.,Morais,Hugo.,Castro, Rui., and Vale, Zita., Evaluation of different initial solution Algorithms to be used in the heuristics optimization to solve the energy resource scheduling in Smart Grids Applied Soft Computing, 2016; (48): 491-506.

[20] Zhao, Qing., Xiong, Congcong., Yu, Ce., Zhang, Chuanlei., and Zhao, Xi., A New Energy-Aware Task Scheduling Method for Data-Intensive Applications in the Cloud, Journal of Network and Computer Applications, 2016; (59): 14-27.

[21] Ismail,Leila.,and Fardoun, Abbas., EATS: Energy-Aware Tasks Scheduling in Cloud computing Systems Procedia Computer Science, 2016; (83): 870 - 877.

[22] Milenkovic, Milan., Operating Systems: Concepts and Design, McGraw-Hill, Inc. New York,1992,ISBN:0-07911365-6.

[23] Silberschatz, Abraham.,Galvin, Peter Baer.,and Gagne, Greg., Operating System concepts, Seventh Edition, 2005, John Wiley and Sons. ISBN: 0-471-69466-5.

[24] William, S., Operating Systems-5 ${ }^{\text {th }}$ edition ISBN: 9780131479548

[25] Douligeris, Christos., and Feng, Gang., Using Hopfield Networks to Solve Assignment Problem and N- Queen Problem: An Application of Guided Trial and Error Technique, I.P. Vlahavas and D. Spyropoulos (Eds.): SETN, LNAI 2308, 2002; Springer-Verlag Berlin Heidelberg. $325-336$.

[26] Zomaya, A.Y. and Lee, Y.C., Energy Efficient Distributed Computing Systems, First Edition, IEEE Computer Society, John Wiley and Sons, USA, 2012, 0138. 